\title{
WAVE INTERACTION WITH LARGE ROUGHNESS ELEMENTS ON AN IMPERMEABLE SLOPING BED
}

\author{
Bjarne Jensen $^{1}$, Erik Damgaard Christensen ${ }^{1}$ and B. Mutlu Sumer ${ }^{1}$
}

\begin{abstract}
The present paper presents the results of an experimental and numerical investigation of the flow between large roughness elements on a steep sloping impermeable bed during wave action. The setup is designed to resemble a breakwater structure. The work is part of a study where the focus is on the details in the porous core flow and the armour layer flow i.e. the interaction between the two flow domains and the effect on the armour layer stability. In order to isolate the processes involved with the flow in the porous core the investigations are first carried out with a completely impermeable bed and successively repeated with a porous bed. In this paper the focus is on the impermeable bed. Results are obtained experimentally for flow and turbulence between the roughness elements on the sloping bed. Numerical simulations have reproduced the experimental results with good agreements and can hereby add more details to the understanding of the fluid-structure interaction.
\end{abstract}

Keywords: Fluid-structure interaction; Breakwaters; Model scale experiments; Numerical modelling

\section{INTRODUCTION}

The breakwater structure is widely used within coastal and harbour engineering for providing sheltering against offshore wave action. Often the breakwater structure is designed as a porous structure which allows a water flow through the structure while the wave energy is removed. The internal porous core can be made of sand and gravel materials with specific gradations. To protect these rather fine grained internal layers from being eroded by the waves one or several layers of larger stones are placed on top of the core material. These are referred to as cover or armour stones.

The scope of the work presented here is to study the stability of the breakwater armour layers with special focus on the interaction between the armour and the porous core material. The flow in and out of the porous core contributes to the stability and/or instability of the armour layers. In general the flow can be divided into two domains: (i) porous flow in the core material and (ii) armour layer flow just above, and in between, the armour layers. The theoretical background for the porous flow was described in Engelund (1954) for both laminar and turbulent flows. In Burcharth and Andersen (1995) the porous flow was investigated with reference to breakwater structures. The armour layer flow has typically been included via model scale experiments where the entire structure including the porous core is constructed in laboratory scale and exposed to wave action. Recent examples on such experiments are Andersen et al. (2011) and Burcharth et al. (2006) where stability and overtopping is investigated. In addition to stability and overtopping also the flow through the porous material of the structure is studied e.g. in terms of pressure distributions through the breakwater. Recent examples of this are Muttray and Oumeraci (2005) and Vanneste and Troch (2012).

The stability is evaluated based on the observed damages during the experiments; however the details on the failure mechanism such as the porous flow and the armour layer flow is not investigated in these types of experiments. Examples of a more detailed approach are seen in Torum (1994) where forces have been measured on spherical armour units on a sloping breakwater front in laboratory scale. In Hald (1998) forces were measured on real armour stone also in laboratory scale. These studies focused on the response as function of the incoming wave condition whereas details on velocities and turbulence in the armour layer as well as the porous flow were not investigated.

Numerically the entire system can be further investigated which is the case in for example Losada et al. (2005) and recently in del Jesus et al. (2012) and Lara et al. (2012); however due to limitations in computational resources it is still difficult to fully resolve the details of the flow between the armour stones and in the porous core. In Lai et al. (2010) the flow between actual spherical stones were resolved in a numerical model however on a mild sloping beach.

The present work is focused on the details in the porous core flow and the armour layer flow i.e. the interaction between the two flow domains and the effect on the armour layer stability. In order to isolate the processes involved with the flow in the porous core the experiments are first carried out with a completely impermeable bed and successively repeated with a porous bed. In this paper the focus is on the impermeable bed experiments with one layer of roughness elements. With this methodology the complexity of the structure is increased step by step as the experiments are progressing. When adding the different structural parts to the experiments one at a time it is possible to see the effect of the physical processes accounted for by these structural elements.

\footnotetext{
${ }^{1}$ Department of Mechanical Engineering, Technical University of Denmark, DK-2800 Kgs. Lyngby, Denmark
} 


\section{EXPERIMENTAL SETUP AND CONDITIONS}

\section{Facility and Setup}

All experiments were carried out in a wave flume at the hydraulic laboratory at DTU. The flume has a length at $25 \mathrm{~m}$, a width at $0.6 \mathrm{~m}$, and a depth at $0.8 \mathrm{~m}$. The water depth for the present experiments was fixed at $0.4 \mathrm{~m}$. The flume is equipped with a piston-type wave maker in one end for generating regular as well as irregular wave conditions. In the opposite end the flume is equipped with a parabolic shaped wave absorber. At the general testing area the sides of the flume is made out of transparent glass which enables a visual observation of the experiments as well as laser LDA measurements from the side. An overview of the entire flume setup is shown in figure 1.

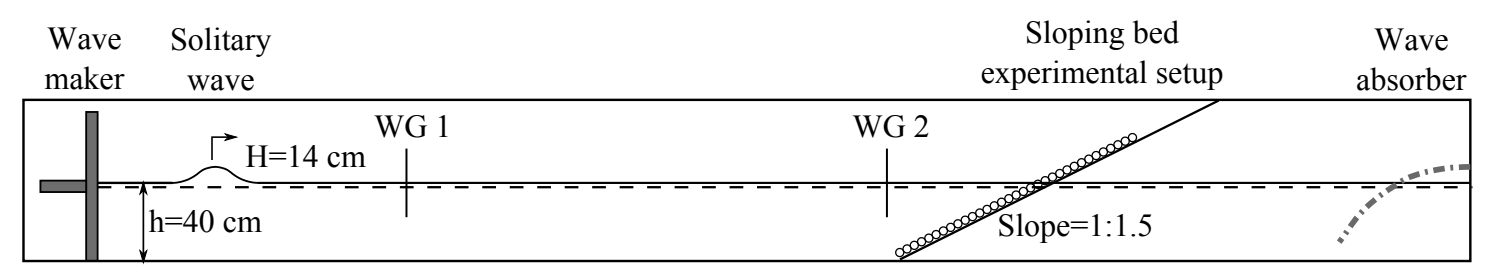

Figure 1: Sketch of the experimental setup for the impermeable bed experiments with smooth bed and roughness elements.

The slope used for the rough bed experiments was arranged with an inclination of 1:1.5. The bed was made out of a plastic PVC plate with a thickness at $20 \mathrm{~mm}$ and a length at $1.5 \mathrm{~m}$. The width corresponds to the width of the flume at $0.6 \mathrm{~m}$. The plate was supported on the top of the flume by a steel profile spanning over the flume in the transverse direction. Furthermore the plate was fixated at the bottom of the flume to ensure that there was no movements of the bed during wave action. The interface between the flume walls and the sides of the sloping bed was sealed with silicon filler to ensure that no water exchange took place between the front and the back of the sloping bed. Water where pumped to the rear side of the sloping bed before start of the experiments. A general sketch of the rigid bed model is shown in figure $2 \mathrm{a}$. The sloping bed was covered with an idealized armour layer consisting of spherical plastic elements with a diameter of $D=38 \mathrm{~mm}$ glued to the bed in a 90 degree arrangement, see figure $2 \mathrm{~b}$. The plastic spheres were applied in one layer.

In addition to the above described rough bed experiments a series of smooth bed experiments has been conducted as well. These will only be included briefly in the present paper. The setup for the smooth bed experiments was identical to the rough bed experiments only the bed being smooth. Hereby the hydrodynamics accounted for by the sloping bed only can be investigated without the effect of the roughness elements.

A) Section view

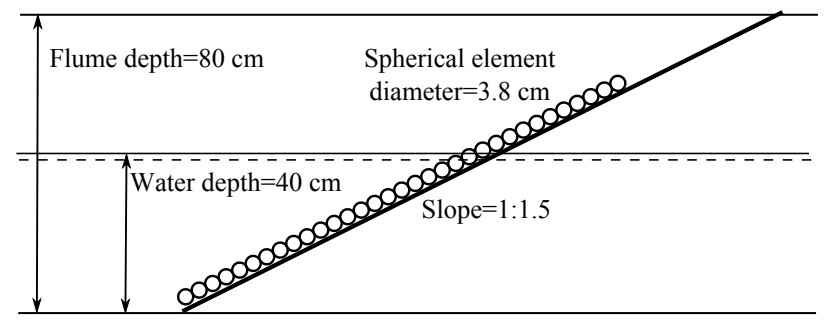

B) Plan view

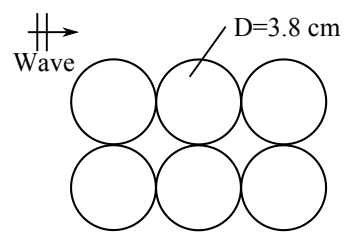

Figure 2: Rigid bed model with spherical plastic balls arranged in a $90^{\circ}$ pattern. 


\section{Test Conditions}

The experiments were performed with a solitary wave which allows for an idealized investigation of the dynamics within one wave cycle including approach, run-up, and run-down. Other studies have previously applied this methodology in order to study the run-up and run-down processes. Examples are Grilli et al. (1994) where solitary wave breaking induced by a breakwater was investigated, Sumer et al. (2011) who studied flow and sediment transport due to a plunging solitary wave, and Lara et al. (2012) who applied a solitary wave for investigating wave interaction with a breakwater both experimentally and numerically.

The offshore water depth was $h=40 \mathrm{~cm}$ for all experiments and the height of the solitary wave was $H=14 \mathrm{~cm}$. The undisturbed offshore surface elevation is given by the small-amplitude solitary wave theory as:

$$
\eta=H \operatorname{sech}^{2}(\omega t)
$$

where $H$ is the hight of the solitary wave measured from the still water level, $t$ is time, and $\omega$ is given as:

$$
\omega=\sqrt{\frac{3}{4} g H} \frac{1}{h}
$$

where $g$ is the acceleration due to gravity. Similar to sinusoidal waves a time scale can be defined by:

$$
T=\frac{2 \pi}{\omega}=2 \pi \sqrt{\frac{4}{3 g H}} h
$$

which can be interpreted as the time scale characterizing the width of the surface elevation time series as described in Sumer et al. (2011). This quantity was $T=2.48 \mathrm{~s}$ in all experiments. The experiments were performed at a Keulegan-Carpenter number at $K C=45\left(=U_{m} T_{w} / D\right)$ where $U_{m}$ is the maximum bed parallel velocity, and a corresponding Reynolds number at $R e=4 \times 10^{4}\left(=D U_{m} / v\right)$.

The sampling frequency of the measurements was $120 \mathrm{~Hz}$. The number of runs for each measuring point (for ensemble averaging) was 30 for the impermeable rough bed experiments. A sensitivity analysis carried out indicated that the statistical quantities, the mean values and the standard deviations, converged to constant values for these sample sizes as shown in figure 3. Here the maximum ensemble averaged RMS value of the fluctuating component of the velocity is depicted against the number of repetitions applied for the ensemble averaging. The left panel shows the error in terms of the absolute RMS value normalised by the mean value of the ensemble averages for 25-30 repetitions. The right panel shows the absolute RMS values.
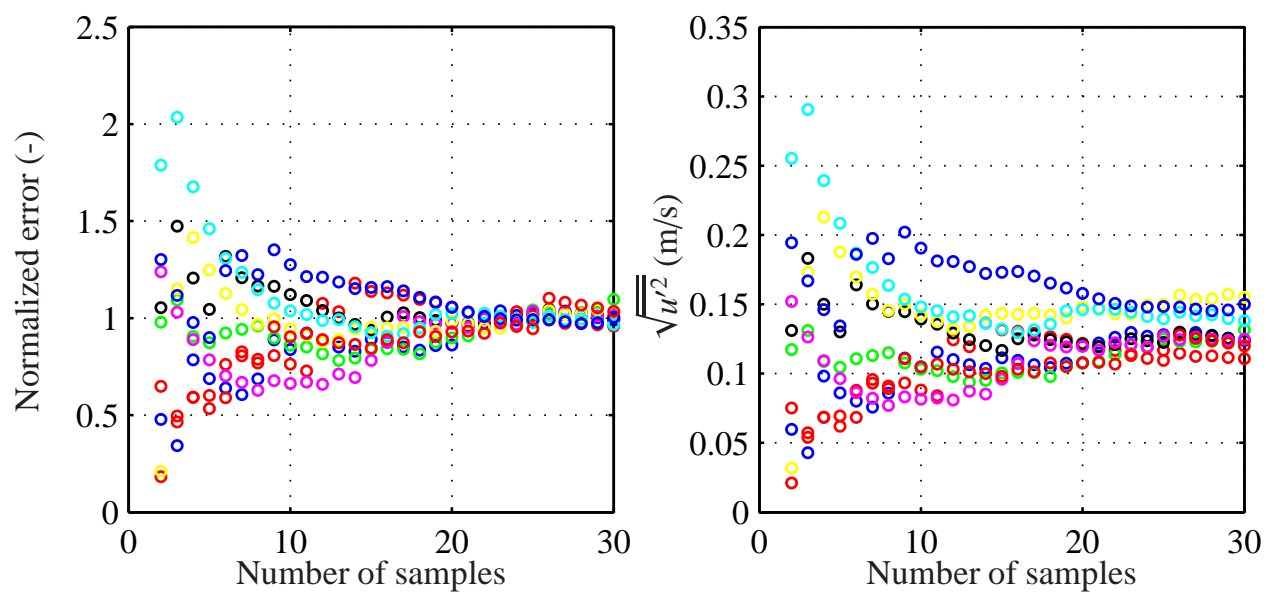

Figure 3: Sensitivity analysis for convergence of RMS values as function of sample size.

The RMS values shown in figure 3 is also applied to quantify the turbulence level in terms of the RMS value of the fluctuating component of the velocity, $u^{\prime}=u-\bar{u}$. This is found as:

$$
\sqrt{\overline{u^{\prime 2}}}(t)=\left\{\frac{1}{N-1} \sum_{i=1}^{N}\left\{[u(t)]_{i}-\bar{u}(t)\right\}^{2}\right\}^{1 / 2}
$$


where $N$ is number of repetitions.

The waves were found to be reproducible. Twenty arbitrary selected time series of the surface elevation at the toe of the sloping bed were plotted together. Here it was seen that they collapsed on a single curve, which confirmed the repeatability of the generated wave.

Regarding the characteristics of the solitary wave the breaking criterion given in Grilli et al. (1997) can be applied. Here the breaking is defined based on the slope parameter, $S_{0}$, defined by:

$$
S_{0}=1.521 \frac{s}{\sqrt{H / h}}
$$

The breaking types are characterized in Grilli et al. (1997) as spilling $\left(S_{0}<0.025\right)$, plunging $(0.025<$ $\left.S_{0}<0.30\right)$, and surging $\left(0.30<S_{0}<0.37\right)$. For the present experimental conditions the slope parameter takes the value $S_{0}=1.71$ which falls outside the defined breaking criterion thus giving a reflecting wave. This is due to the steep sloping bed. As will be seen later a breaking bore/hydraulic jump forms during the run-down.

It is noted that two processes in ordinary oscillating waves are missing in the present idealized solitary wave case, namely the process controlling the wave setup and the process controlling the water table in the porous core of the structure. The latter is of no importance for the present experiments as the bed is impermeable.

\section{Instrumentation and Measurements}

Two types of measurements were performed: velocity measurements and surface elevation measurements. Measurements of velocities and turbulence were performed with Laser Doppler Anemometry (LDA). A DANTEC two-component LDA system was applied in back-scatter mode where the two velocity components, horizontal and vertical, were measured simultaneously. The arrangement of the LDA system is shown in figure 4 for the rigid bed setup with one layer of spherical elements. The velocity is measured in the pore between the flume wall and the two neighbouring spheres across the vertical. Hence the velocity does not represent that measured in a regular pore. However, the effect of the wall has been investigated by repeating a reference experiment with measurement at a larger distance from the wall. This showed no wall effect compared to the pore measurements near the wall. The wall pore is chosen as it gives a greater flexibility in term of positioning the LDA measuring point.

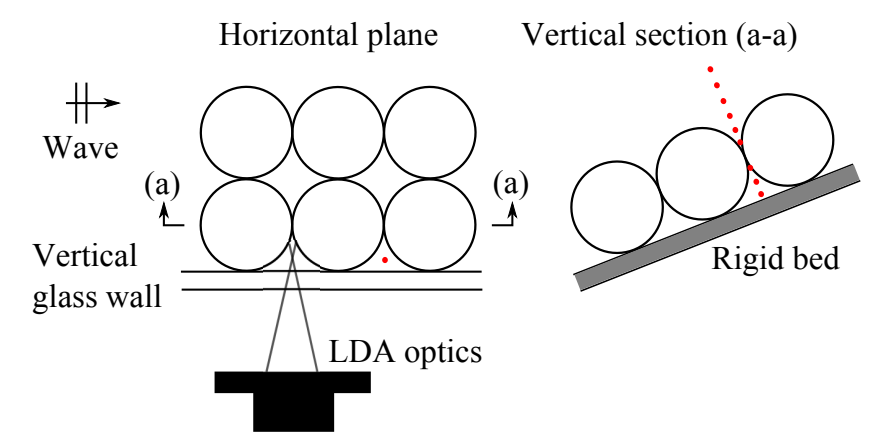

Figure 4: Arrangement of LDA measurements for the rigid bed model with one layer of spherical plastic balls. Measuring points are indicated with dots.

The surface elevation measurements were performed at two locations: at the offshore location and at the toe of the sloping bed as shown in figure 1, WG1 and WG2 respectively. Conventional resistance type wave gauges were used in the measurements. The LDA measurements and the surface elevation measurements were synchronized. In addition to the above, synchronized flow visualization were performed using a digital video recorder applying $250 \mathrm{fps}$. From here the detailed observations are drawn of the entire process of run-up, run-down, breaking and trailing waves.

\section{EXPERIMENTAL RESULTS}

The experiments cover three types of measurements: LDA velocity measurements, surface elevation measurements and video visualization. In the following the main results of the experimental investigations are presented. First, a description of the run-up and run-down cycle is given based on visualisations of the 
experiments. Here the different flow regimes are outlined. Second, the details of the flow is investigated in terms of velocities and turbulence based on the LDA measurements.

\section{Wave Run-up and Run-down Cycle}

The complete cycle of run-up and run-down is visually depicted and described based on high-speed video recordings. Here the surface elevation is schematically presented for a number of relevant time steps during the process. The entire cycle is divided into the following four regimes. i) approach, ii) run-up, iii) run-down and, iv) secondary run-up.

Figure 5 shows the entire sequence of run-up, run-down and trailing wave with secondary run-up. The wave has already been characterised as being reflective i.e. no breaking takes place during the run-up. In Grilli et al. (1997) and Jensen et al. (2003) the run-up phase is divided into several types of flow regimes depending on either the steepness of the sloping bed or the amplitude of the wave. At the lower part of the slope the thickness of the run-up wedge may be several times the roughness of the armour layer. Here the flow has similarities with a rough bottom channel flow. At the upper part of the slope the run-up wedge thickness is less than the roughness, which may resemble flow around obstacles as also described in Andersen et al. (2011). The effect of the roughness elements on the bed is clearly seen at the front of the upper surface wedge. This shows a highly disturbed and turbulent flow due to the flow around the roughness elements. Furthermore the front part of the flow generates an aeration zone where a large amount of air is trapped and released from in-between the roughness elements as the surface front moves up along the slope.

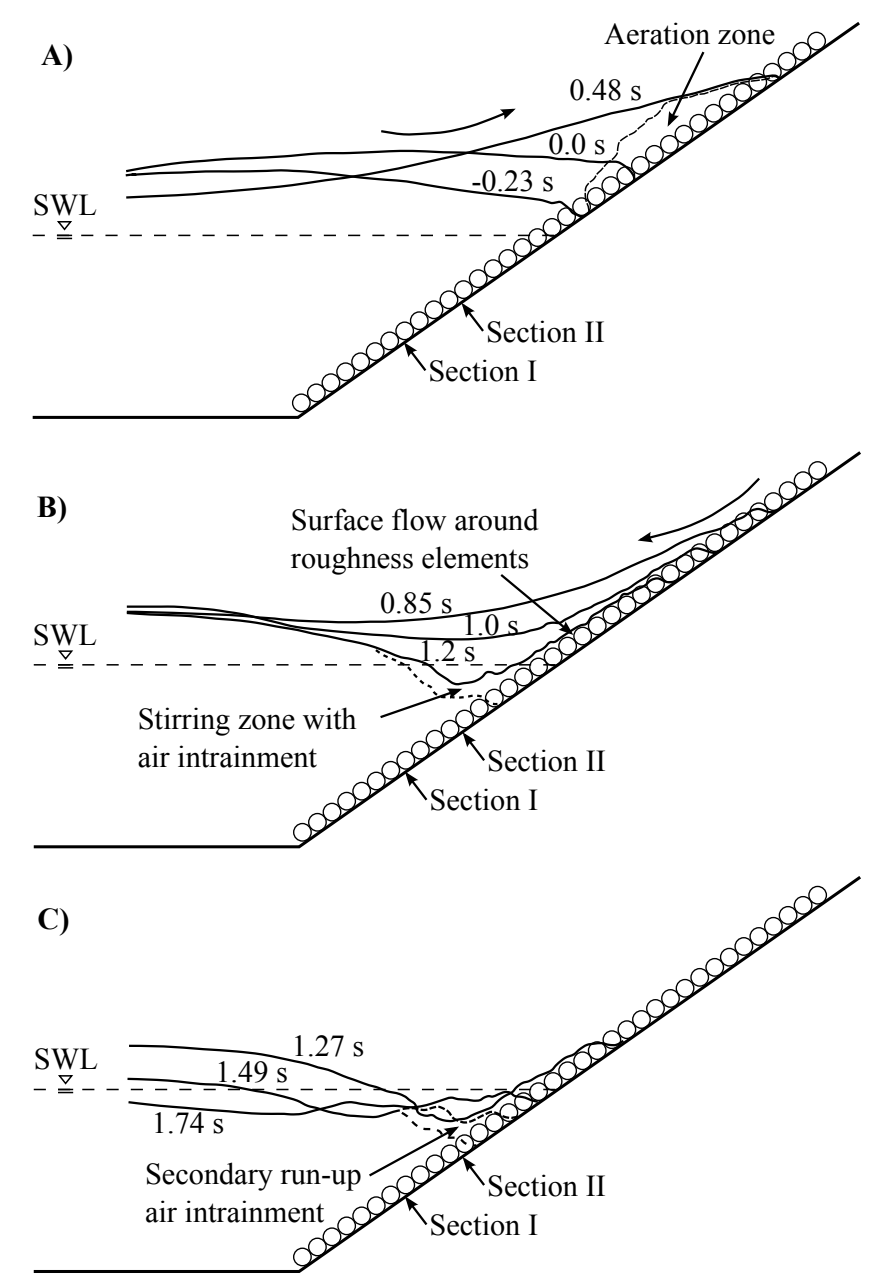

Figure 5: Visualisation of surface elevation on rough bed during A) run-up, B) run-down, and C) trailing wave.

Following maximum run-up the flow reverses and initiates the run-down phase. The upper part of the slope experiences a flow where the relatively low water depth is maintained. A hydraulic jump is seen at 
the transition between the upper part of the flow (low water depth with supercritical flow) and the lower part (higher water depth with subcritical flow). At the end of the run-down phase the downwards directed flow interacts with the volume of water above the lower part of the slope and generates a breaking bore. The breaking process on the run-down is also described in Jensen et al. (2003) and is shown numerically and experimentally in Pedersen and Gjevik (1983).

\section{Velocity and Turbulence}

Figure 7 shows the bed-parallel-velocity time series. The flow direction above the spheres is calculated based on the measured parallel and normal velocities, $u$ and $v$ and are shown in the top panel of figure 7. Here the flow reversal from run-up to run-down is clearly seen. The definition of the flow direction is given in figure 6. Fluctuations appear in the run-up phase (B in figure 7) and throughout the entire wave cycle as a result of locally generated turbulence around the spheres. Close to the bed just above and in between the spherical plastic elements the velocity is highly effected by the presence of the spheres which is also seen in terms of a high level of fluctuations throughout the wave cycle. The free stream fluctuations as well as the fluctuations around the spheres indicates one or several contributions to the turbulence during the run-up and partly the run-down phase which can be accounted for by the roughness elements. However the additional turbulence due to the breaking bore during run-down is also seen (A in figure 7). Also turbulence generated due to the turbulent boundary layer on the impermeable bed will be present however this may be difficult to distinguish from the turbulence generated due to the roughness elements. Later in this paper a comparison is given to smooth bed experiments where the effect of the roughness is removed.

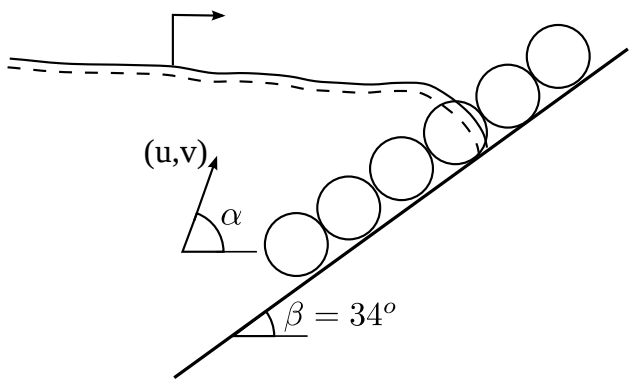

Figure 6: Definitions sketch of flow angle, $\alpha$, calculated based on parallel and normal velocities, $u$ and $v$. Angle of the sloping bed is $\beta=34^{\circ}$.

The turbulence levels presented in figure 8 further show the effect of the roughness elements as already mentioned. Again, the flow direction is shown in the top panel and follow the definition in figure 6 . The turbulence production is initiated from the beginning of the run-up phase and grows gradually until the point where the breaking bore during the run-down phase generates a peak increase in the turbulence level. During run-up fluctuations are seen both in the free stream as well as in the pores. These may originate from two different processes namely the boundary layer turbulence above the roughness elements and wake turbulence formed locally in-between the roughness elements.

Above the roughness elements the run-up turbulence develops to a smaller level than in-between the roughness. At this point (B in figure 8) the turbulence is mainly generated by boundary layer turbulence above the roughness elements as well as lee wake turbulence from in-between the roughness elements which is diffused up into the upper layers. A large increase is seen when the run-down breaking occurs (A in figure 8). Above the roughness elements this process is seen earlier than in-between the roughness. Below the top of the roughness but above the center of the roughness a larger turbulence level is seen ( $\mathrm{C}$ in figure 8). Here the effect of lee wake turbulence from the roughness elements is seen. Also it is clear how the turbulence levels drop momentarily at the point of flow reversal from run-up to run-down. 


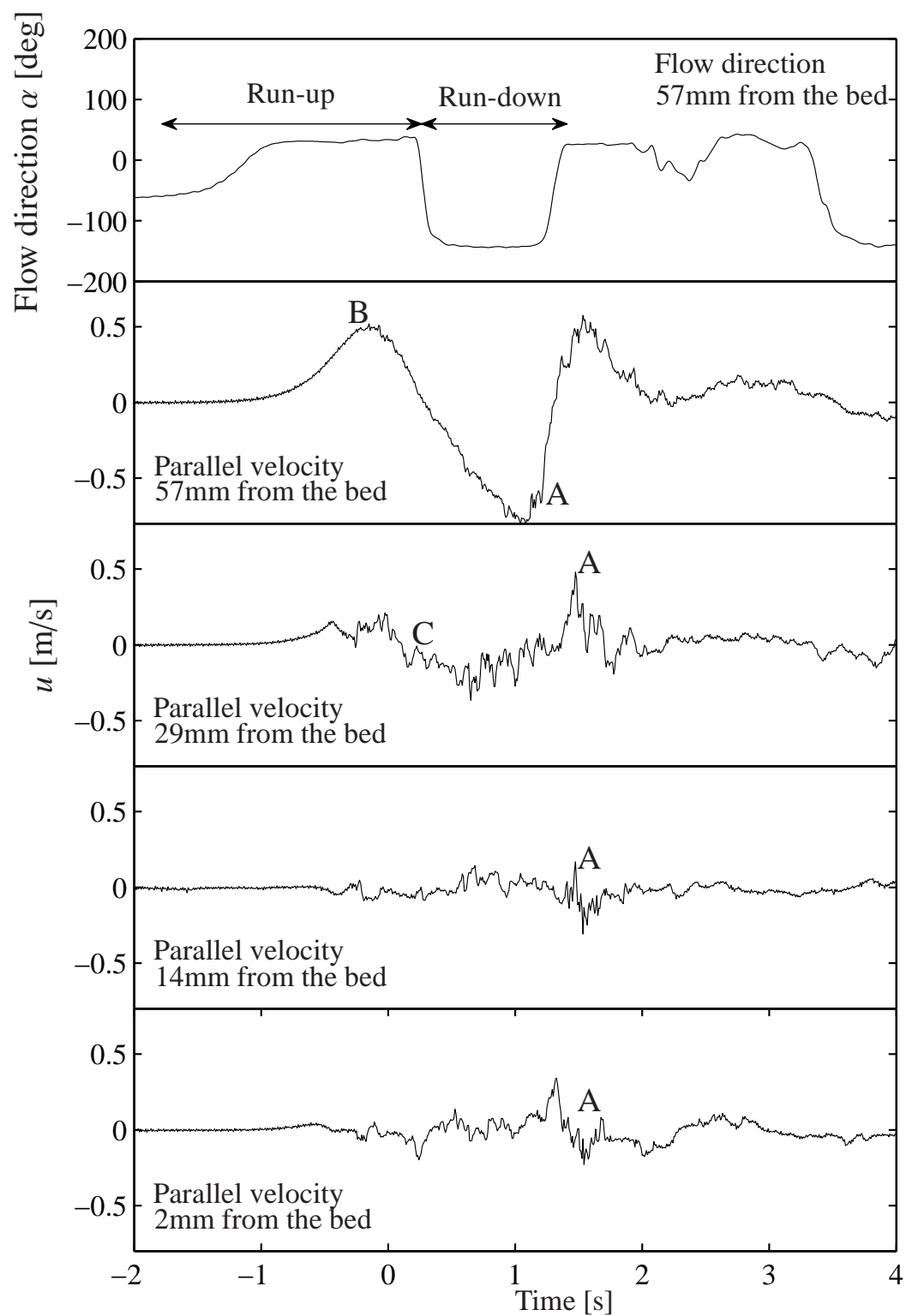

Figure 7: Time series for velocities parallel to the bed for solitary wave at measuring section I on rough bed with one layer of spheres. The flow direction in the free stream point above the spheres is shown as a reference signal in the top panel. Notation A, B and C refers to the discussion in the text. 


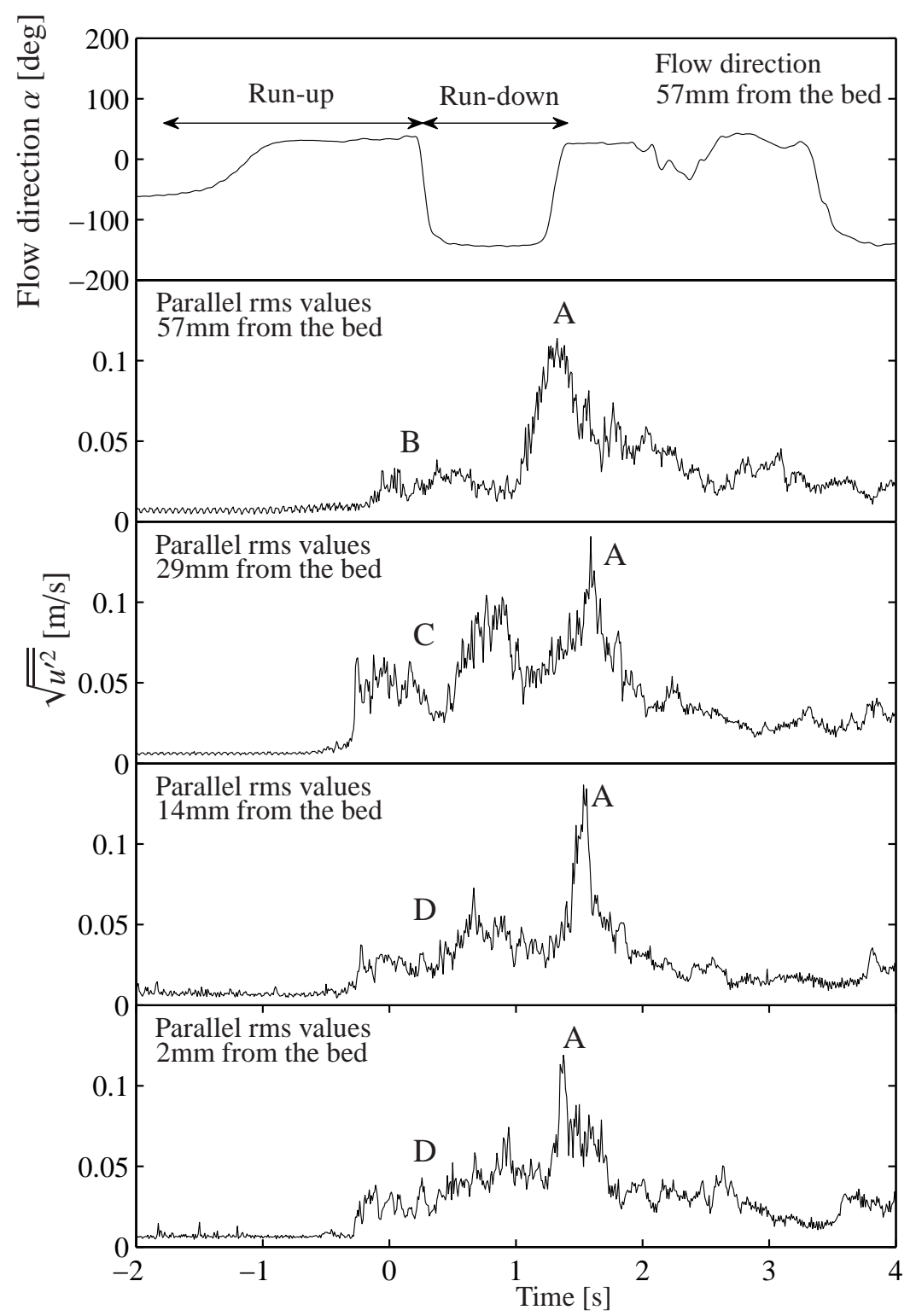

Figure 8: Time variation of the RMS values of the fluctuating component of the velocity component parallel to the bed at measuring section I on rough bed with one layer of spheres. The flow direction in the free stream point above the spheres is shown as a reference signal in the top panel. Notation $A, B, C$ and $D$ refers to the discussion in the text.

Finally a brief comparison is given with the corresponding data performed with the smooth sloping bed setup. When the results from the rough bed experiments are compared to identical smooth bed experiments the effect of the surface roughness can be seen. The comparison is summarised in figure 9. Here it is seen how the bed parallel velocities experiences very little fluctuations during run-up and most of the run-down phase for the smooth bed experiments. At the end of the run-down phase fluctuations are initiated first due to boundary layer turbulence and immediately after further increased due to the breaking bore which is very pronounced for the smooth bed experiments. Compared to the rough bed experiments it is seen how the turbulent fluctuations starts to develop during the run-up phase caused by locally generated turbulence inbetween the spherical elements. Again a large peak in the fluctuations are found at the end of the run-down phase where the breaking bore transports turbulence down to the measuring section. Furthermore it is seen how the flow reverses from run-down to secondary run-up at an earlier stage for the rough bed compared to smooth bed. 


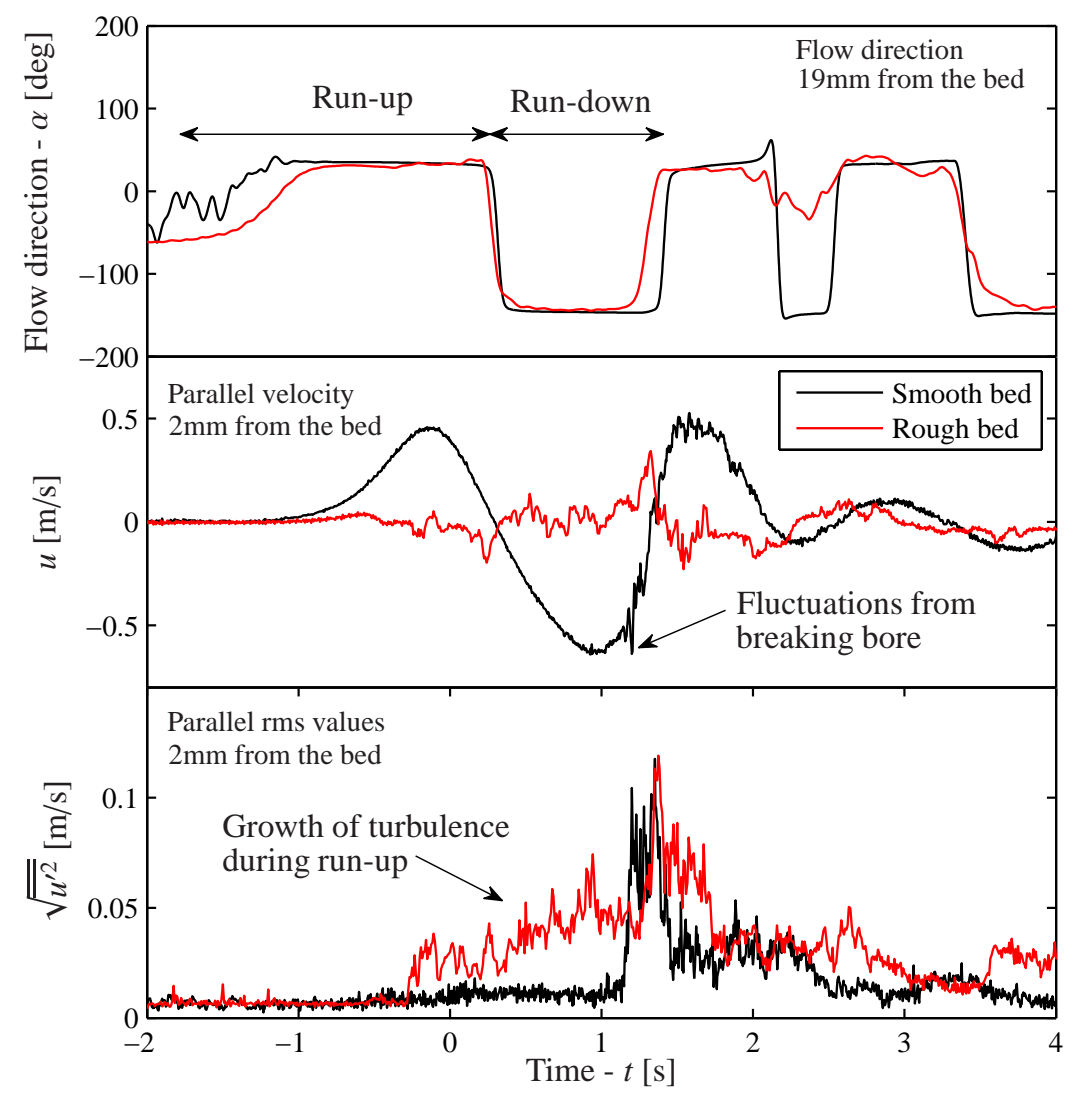

Figure 9: Time series for velocities and RMS values parallel to the bed for solitary wave at measuring section I. Comparison of smooth bed and rough bed with one layer of spheres. The flow direction in the free stream point above the spheres is shown as a reference signal in the top panel.

\section{NUMERICAL MODEL AND SETUP}

The experimental setup has been further investigated by numerical simulations. The open source CFD library OpenFOAM $®$ has been applied including Large Eddy Simulation (LES) turbulence modelling. A detailed model has been setup where the roughness elements on the sloping bed are resolved directly in a periodic domain. The measured free stream flow from the physical experiments is applied as boundary conditions hereby enabling a direct comparison of the simulated and measured results. The numerical model and the model setup is described in the following.

\section{Numerical Model}

The numerical model is based on a finite volume discretisation of the Navier-Stokes equations on a collocated grid arrangement. The Navier-Stokes equations consists of the continuity and momentum equation as follows:

Continuity equation:

$$
\frac{\partial u_{i}}{\partial x_{i}}=0
$$

Momentum equations:

$$
\rho \frac{\partial u_{i}}{\partial t}+\rho u_{j} \frac{\partial u_{i}}{\partial x_{j}}=-\frac{\partial p}{\partial x_{i}}+\frac{\partial}{\partial x_{j}} \mu\left(\frac{\partial u_{i}}{\partial x_{j}}+\frac{\partial u_{j}}{\partial x_{i}}\right)
$$

where $\rho$ is the density of the fluid, $u_{i}$ is the velocity vector $u_{i}=(u, v, w), p$ is the pressure, $\mu$ is the dynamical viscosity, $t$ is the time, and $x$ is the spatial variable.

For high Reynolds number flows the turbulent fluctuations may not be resolved directly by the computational grid. Therefore a turbulence model must be introduced to account for the effects of the turbulent fluctuations. For the present simulations a Large Eddy Simulation (LES) model has been applied that allows 
for a direct simulation of the large scale turbulent fluctuations while the LES model adds the effect of the small scale turbulent fluctuations. LES modelling includes the direct simulation of turbulent fluctuations larger than the selected filter scale however this also sets some strict requirements to the grid resolution. In general a finer grid resolution is required compared to RANS turbulence models however some applications of LES within coastal engineering has been seen e.g. Christensen and Deigaard (2001) and Christensen (2006) applied LES modelling for investigation of spilling and plunging breakers.

The LES model is based on a spatial filtering of the Navier-Stokes equations. A top-hat filter is applied where the computational grid is used as the filter. The filtered Navier-Stokes equation reads:

$$
\rho \frac{\partial \bar{u}_{i}}{\partial t}+\rho \frac{\partial \overline{u_{i} u_{j}}}{\partial x_{j}}=-\frac{\partial \bar{p}}{\partial x_{i}}+\frac{\partial}{\partial x_{j}} \mu\left(\frac{\partial \bar{u}_{i}}{\partial x_{j}}+\frac{\partial \bar{u}_{j}}{\partial x_{i}}\right)
$$

where overbar denotes a filtered quantity. In equation 8 the second term on the left hand side is split up into two terms as:

$$
\frac{\partial \overline{u_{i} u_{j}}}{\partial x_{j}}=\frac{\partial \bar{u}_{i} \bar{u}_{j}}{\partial x_{j}}+\left(\frac{\partial\left(\overline{u_{i} u_{j}}-\bar{u}_{i} \bar{u}_{j}\right)}{\partial x_{j}}\right)
$$

The first term on the right hand side is simulated directly while the second term is moved to the right hand side of equation 8 and must be modelled. This term is also referred to as the sub-grid scale Reynolds stress:

$$
\tau_{i j} s=-\rho\left(\overline{u_{i} u_{j}}-\bar{u}_{i} \bar{u}_{j}\right)
$$

Equation 10 is called the closure problem for which a model must be applied. This model will be referred to as a sub-grid scale model (sgs model). For the present simulations the Smagorinsky sgs model is applied. The sub-grid scale stresses given in equation 10 are modelled as:

$$
\begin{array}{r}
\tau_{i j} s=\frac{1}{3} \tau_{k k} s \delta_{i j}=2 \mu_{t} \bar{S}_{i} j \\
\bar{S}_{i j}=\frac{1}{2}\left(\frac{\partial \bar{u}_{i}}{\partial x_{j}}+\frac{\partial \bar{u}_{j}}{\partial x_{i}}\right)
\end{array}
$$

Here $\mu_{t}$ is the eddy viscosity which is found as:

$$
\mu_{t}=\rho\left(C_{s} \Delta\right)^{2}|\bar{S}|
$$

where $\Delta$ is the filter length scale and $|\bar{S}|=\left(2 \bar{S}_{i j} \bar{S}_{i j}\right)^{1 / 2}$. $C_{s}$ is the Smagorinsky constant that is generally in the order of 0.065 to 0.2 . For the present simulations it is set to 0.1 however the optimal value can vary from case to case.

\section{Model Setup}

The model has been setup to reproduce the experimental results obtained for one layer of spherical roughness elements on the sloping impermeable bed. Currently the focus has been on the detailed flow around the spherical elements. Therefore a model has been setup that includes the local area around the spheres. Only one spherical elements has been resolved and periodic boundary conditions has been applied in order to add the effect of multiple elements. With this approach the oscillating flow around the roughness element is modelled; however the free surface is not included. This leaves out some effects such as wave breaking as will be seen later.

Figure 10 presents the setup in terms of geometry and applied boundary conditions. The domain has horizontal dimensions corresponding to the diameter of one sphere, $D=38 \mathrm{~mm}$ and a hight at $3 \mathrm{D}$. 


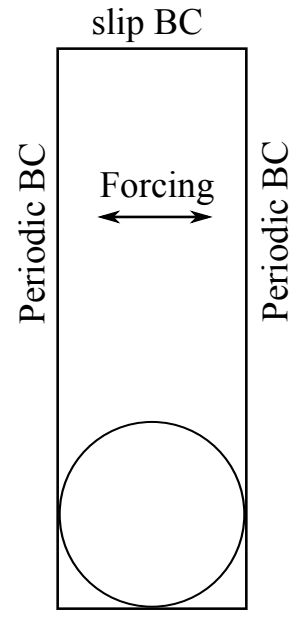

Wall BC

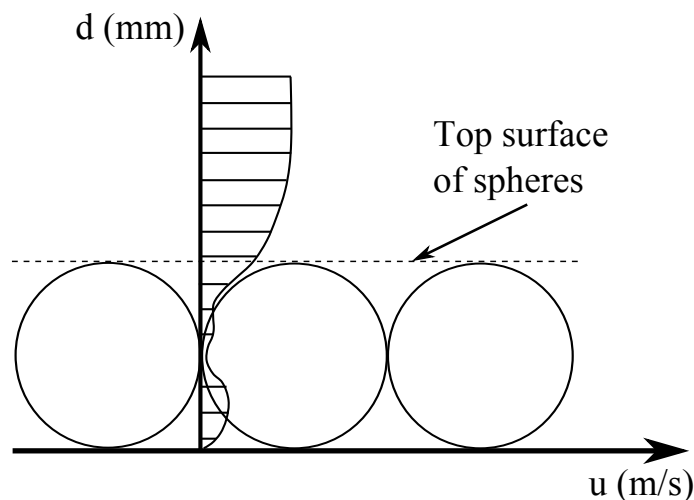

$\mathrm{u}(\mathrm{m} / \mathrm{s})$

Figure 10: Numerical model setup. One spherical element in a periodic domain.

At the bottom below the sphere a wall boundary condition is applied where $u=0$ at the boundary. The surface of the sphere is modelled with a wall boundary condition as well. A slip boundary condition is applied at the top of the model while a periodic boundary condition is applied on the vertical sides of the model. Hereby any quantity transported out of the domain e.g. via the right hand boundary will at the same time be moved into the domain via the left hand boundary. This will create the effect of an infinite number of spheres placed next to each other.

The flow is driven by a forcing term in the momentum equation that is based on the experimental measurements. Here the velocity measurement from the free stream region above the spheres (one run-up and run-down cycle) are used as input. The momentum equation (7) is extended as:

$$
\rho \frac{\partial u_{i}}{\partial t}+\rho u_{j} \frac{\partial u_{i}}{\partial x_{j}}=-\frac{\partial p}{\partial x_{i}}+\frac{\partial}{\partial x_{j}} \mu\left(\frac{\partial u_{i}}{\partial x_{j}}+\frac{\partial u_{j}}{\partial x_{i}}\right)+f_{i}
$$

where the forcing term, $f_{i}$, is included as:

$$
f_{i}=\rho \frac{\partial u_{e i}}{\partial t}
$$

where $u_{e i}$ is the experimentally measured velocity vector $u_{e i}=\left(u_{e}, v_{e}, w_{e}\right)$.

The computational grid is setup with the smallest grid cell being $0.3 \times 0.3 \times 0.3 \mathrm{~mm}$ (at the surface of the sphere) and the largest grid cell being $2 \times 2 \times 4 \mathrm{~mm}$ (at the top of the model). A total of 800,000 grid cells were applied. The computational grid is presented in figure 11. Parallel processing was applied where the model domain was decomposed into 6 domains. 

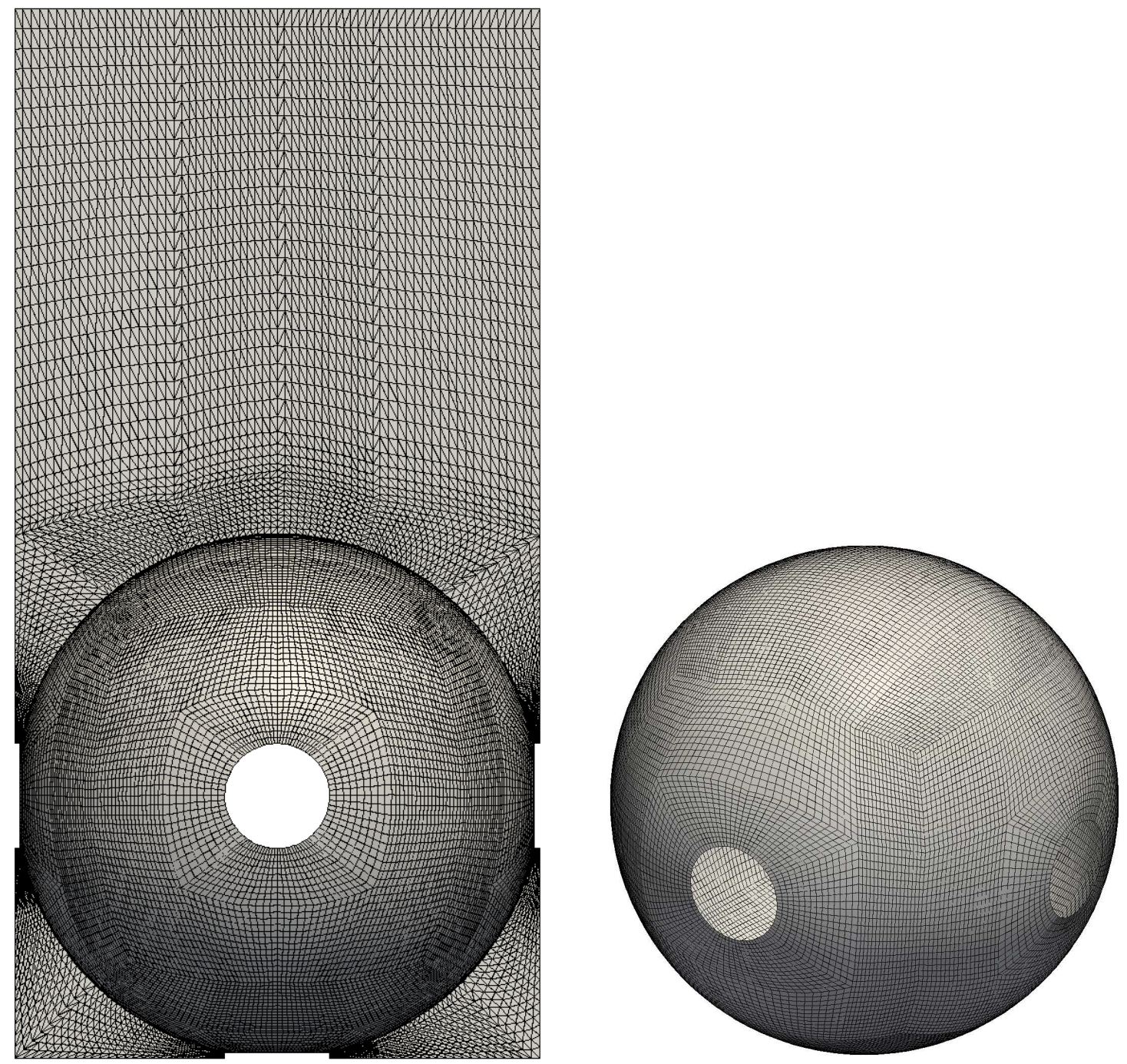

Figure 11: Computational grid. Left panel: full view of domain. Right panel: close up on spherical surface.

\section{NUMERICAL RESULTS}

The numerical results have been compared to the experimental measurements previously presented. The bed parallel velocity is presented in figure 12 as ensemble averaged vertical velocity profiles. Time steps are selected to cover the entire cycle of run-up, run-down and secondary run-up. Measurements (black line with circles) are compared to the numerical results (red line).

The model is seen to capture the measurements with good agreement during the run-up and most of the run-down phase. Both the flow above the spheres as well as in the pores in-between the spheres are well described. During the last part of the run-down and especially at the secondary reflected run-up some deviations are seen. This might be due to the fact that the free surface is not included in the model and thereby the breaking bore during the run-down is not simulated. As seen for the experimental results in figure 7 and figure 8 the flow is clearly affected by the run-down breaking in terms of velocities and fluctuations. This effect is not represented in this numerical model.

Figure 13 shows an iso-surface plot of vorticity around the longitudinal direction (in-line with the flow direction) and the transversal direction. On the left hand panel it is seen how the flow separates on the surface of the sphere and four symmetrical vortices are formed which travels downstream with the flow. These vortices have similarities with horse-shoe vortices which are well known from e.g. flow around vertical cylinders. On the right hand panel it can be seen how a boundary layer develops both on the smooth bed below the sphere as well as above the sphere. On the down stream side of the spheres a lee zone is apparent where the boundary layer does not develop. 

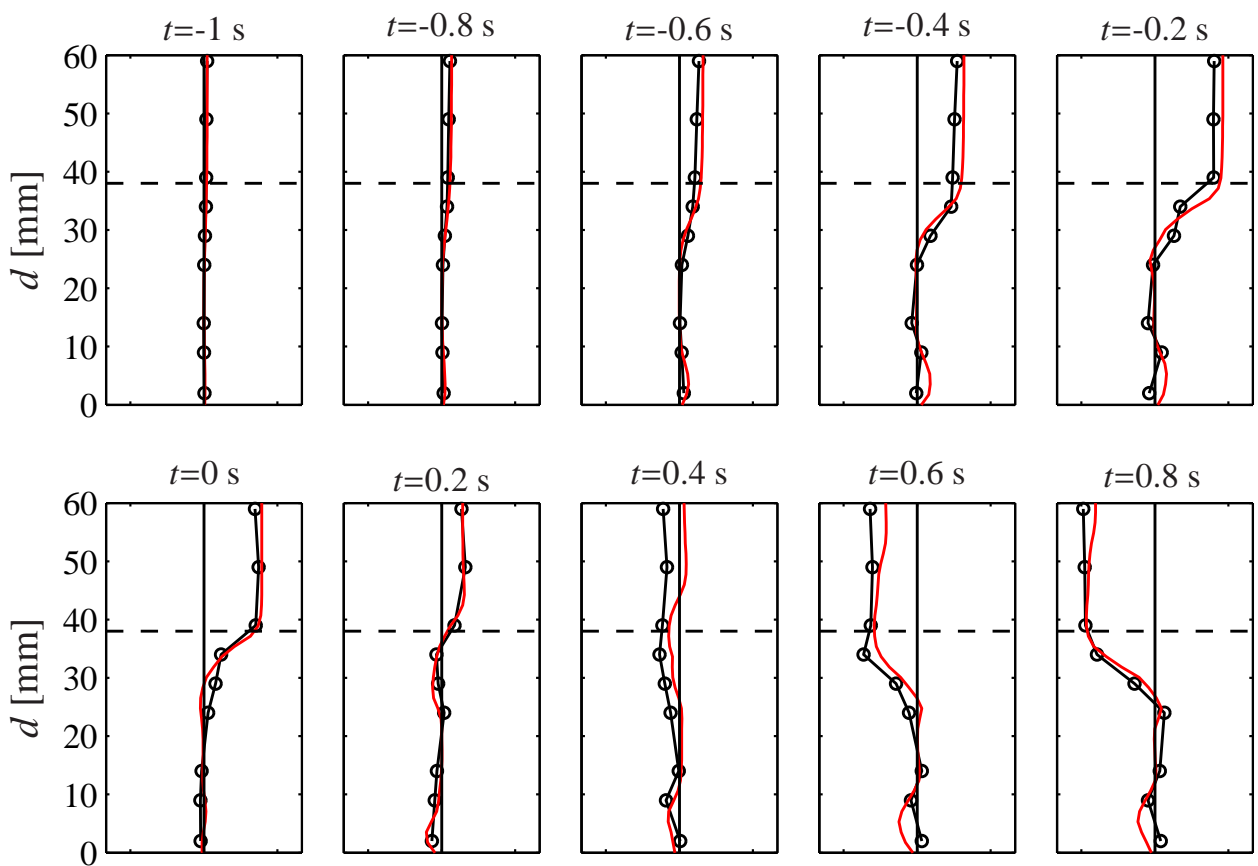

$t=0.4 \mathrm{~s}$
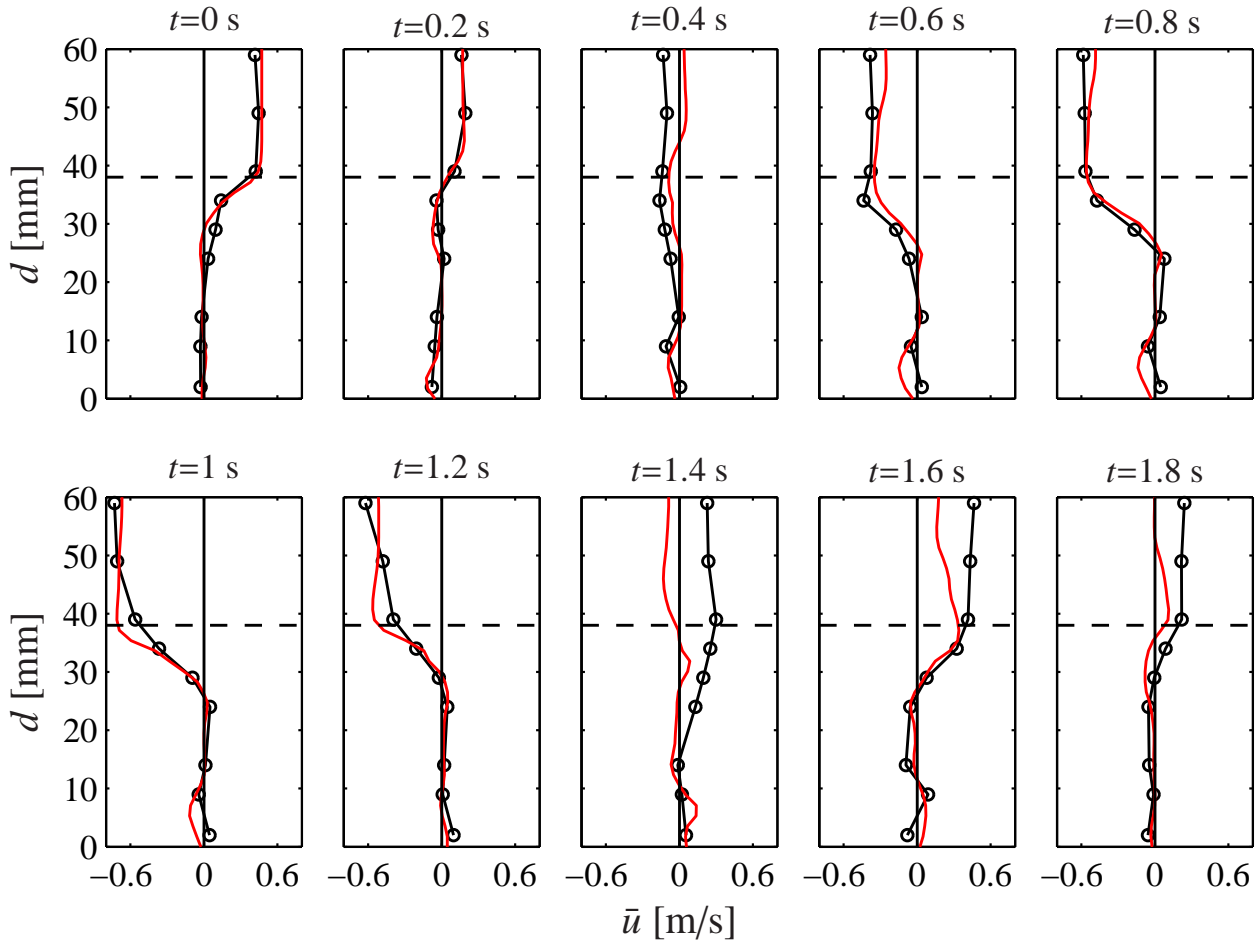

Figure 12: Ensemble averaged velocity profiles for velocities parallel to the bed for solitary wave at measuring section I. Comparison of measurements (black line with circles) and simulated results (red line) for rough bed with one layer of spheres. The horizontal dashed line shows the top surface of the spheres. $t=-1-0.2 \mathrm{~s}$ corresponds to the run-up phase and $t=0.4-1.2 s$ corresponds to the run-down phase
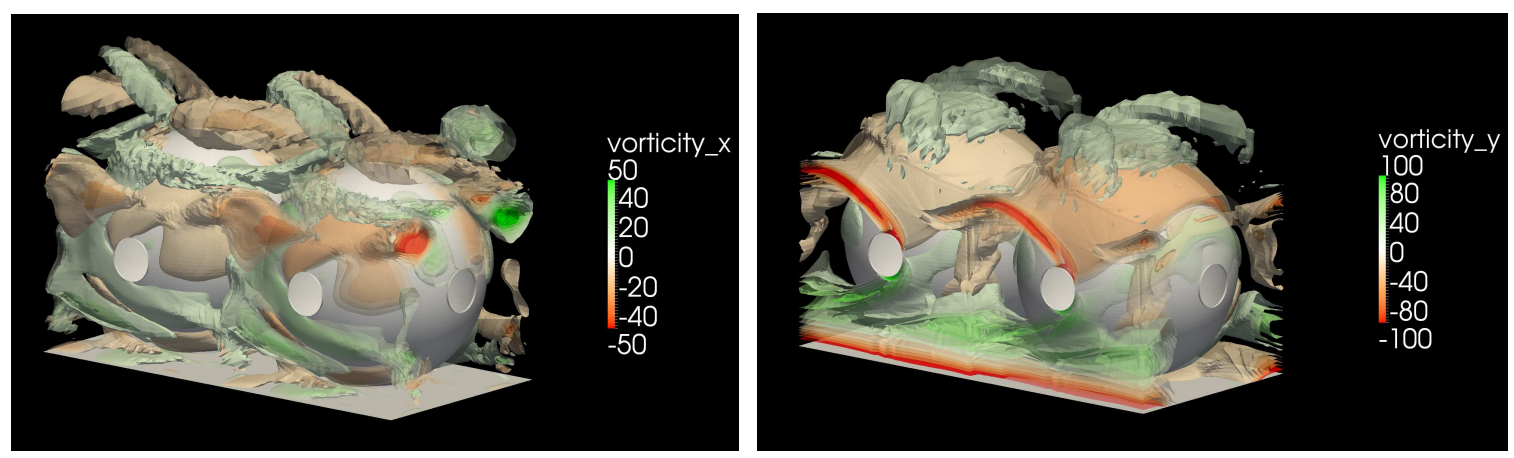

Figure 13: Iso-surface of vorticity at a phase angle of $\omega t=30 \mathrm{deg}$. Left panel: vorticity around the longitudinal direction (X-axis). Right panel: vorticity around the transversal direction (Y-axis). 


\section{CONCLUSION}

Experiments have been conducted with an impermeable sloping bed with a structured layer of spherical roughness elements. The setup resembles a simplified breakwater structure. The experiments are part of a series of several experimental investigations where the individual physical processes are described. This is achieved by starting out with a very simple setup where the different structural parts are added; one at a time. Hereby the physical processes can be seen and distinguished from each other.

The experiments showed the effect of the roughness elements in terms of the turbulence generation during run-up both locally between the roughness elements and above the roughness as boundary layer turbulence. The breaking mechanism on run-down showed a transport of turbulence below the surface in-between the roughness elements. This process is very clear for the smooth bed experiments however it is also found for the rough bed experiments.

The experiments have been followed by numerical simulations which have been setup to reproduce the experimental results. A periodic domain was applied to simulate the oscillating flow around one spherical roughness element however including the effect of several spheres placed next to each other. Good agreement was found between the simulated and measured ensemble averaged velocities. Some deviations were seen at the very last part of the run-down phase which is explained by the fact that the free surface is not included in the present simulations and thereby the breaking mechanism during the run-down is not described. This effect will be included in future simulations where the free surface is simulated. The numerical results showed a level of details which can be used for in-depth analysis of e.g. erosion mechanisms and armour stone forces.

\section{ACKNOWLEDGEMENTS}

The support of the Danish Ministry of Science, Technology and Innovation through the GTS grant: Fremtidens Marine Konstruktioner (Marine Structures of the Future), is acknowledged.

\section{REFERENCES}

Andersen, T. L., Burcharth, H., and Gironella, X. (2011). Comparison of new large and small scale overtopping tests for rubble mound breakwaters. Coastal Engineering, 58(4):351-373.

Burcharth, H. and Andersen, O. (1995). On the one-dimensional steady and unsteady porous flow equations. Coastal Engineering, 24(3-4):233-257.

Burcharth, H., Kramer, M., Lamberti, a., and Zanuttigh, B. (2006). Structural stability of detached low crested breakwaters. Coastal Engineering, 53(4):381-394.

Christensen, E. (2006). Large eddy simulation of spilling and plunging breakers. Coastal Engineering, 53(5-6):463-485.

Christensen, E. D. and Deigaard, R. (2001). Large eddy simulation of breaking waves. Coastal Engineering, 42(1):53-86.

del Jesus, M., Lara, J. L., and Losada, I. J. (2012). Three-dimensional interaction of waves and porous coastal structures. Coastal Engineering, 64:57-72.

Engelund, F. (1954). On the laminar and turbulent flows of ground water through homogeneous sand. Technical report, Danish Academy of Technical Sciences.

Grilli, B. S. T., Losada, M. A., and Martin, F. (1994). Characteristics of solitary wave breaking induced by breakwaters. Journal of Waterway, Port, Coastal, and Ocean Engineering, 120(1).

Grilli, S., Svendsen, I., and Subramanya, R. (1997). Breaking Criterion And Characteristics For Solitary Waves On Slope. Journal of Waterway, Port, Coastal, and Ocean Engineering, (June):102-112.

Hald, T. (1998). Wave Induced Loading and Stability of Rubble Mound Breakwaters. Series paper no. 18, Aalborg University.

Jensen, A., Pedersen, G. K., and Wood, D. J. (2003). An experimental study of wave run-up at a steep beach. Journal of Fluid Mechanics, 486:161-188.

Lai, J.-W., Hsu, T.-W., and Lan, Y.-J. (2010). Experimental and numerical studies on wave propagation over coarse grained sloping beach. In Coastal Engineering, pages 1-15.

Lara, J. L., del Jesus, M., and Losada, I. J. (2012). Three-dimensional interaction of waves and porous coastal structures. Coastal Engineering, 64:26-46.

Losada, I., Lara, J., Christensen, E., and Garcia, N. (2005). Modelling of velocity and turbulence fields around and within low-crested rubble-mound breakwaters. Coastal Engineering, 52(10-11):887-913.

Muttray, M. and Oumeraci, H. (2005). Theoretical and experimental study on wave damping inside a rubble mound breakwater. Coastal Engineering, 52(8):709-725.

Pedersen, G. and Gjevik, B. (1983). Run-up of solitary waves. Journal of Fluid Mechanics, 135:283-299. 
Sumer, B. M., Sen, M. B., Karagali, I., Ceren, B., Fredsø e, J. r., Sottile, M., Zilioli, L., and Fuhrman, D. R. (2011). Flow and sediment transport induced by a plunging solitary wave. Journal of Geophysical Research, 116(C1):1-15.

Torum, A. (1994). Wave-induced foreces on armor unit on berm breakwaters. Journal of Waterway, Port, Coastal, and Ocean Engineering, 120(3):251-268.

Vanneste, D. and Troch, P. (2012). An improved calculation model for the wave-induced pore pressure distribution in a rubble-mound breakwater core. Coastal Engineering, 66:8-23. 